\title{
L'Analphabetisme des pays industrialises: du mythe a la reconstruction des faits
}

\author{
JEAN-PAUl HAUTECOEUR \\ Institut de l'Unesco pour l'Education
}

\begin{abstract}
SUMMARY.-La primera intención de este texto es relativizar las nociones de analfabetismo y de alfabetismo interpretándolas como hechos ideológicos a situar en una historia que explica su emergencia en lo imaginario de muchas sociedades occidentales.

La segunda intención es descubrir otro espacio de investigación poco frecuentado: las condiciones materiales y sociales del desarrollo del alfabetismo en un contexto histórico particular y el análisis comparado de contextos diferentes como pueden presentar, por ejemplo, los países del Este y del Oeste de Europa.
\end{abstract}

\section{LE MYTHE}

A la question «Quelle est la nature du problème de l'analphabétisme?», un lettré est tenté de répondre: la naturalisation du problème! C'est-à-dire la réduction d'un ensemble de faits de cultura qui concernent l'écrit à un phénomène naturel singulier comparable à une maladie ${ }^{1}$. On associe facilement l'analphabétisme, nouveau et surprenant comme le sida, à une maladie honteuse. En moins sensationnel, on le compare à la cécité, une carence physiologique. Ou encore, à un handicap intellectuel, moteur, comportemental. L'analphabétisme connote un état pathologique du corps physique et psychique. Il appelle un traitement. Propriété médicale.

L'analphabétisme provoque aussi un décollage dans l'imaginaire. On le découvre dans la modernité comme une survivance d'un état antérieur de l'homme -l'enfance naive, préscolaire, dans son environnement maternel- ou comme une survivance d'un état antérieur de l'humanité- le bon sauvage (Eric Havelock fait d'ailleurs remonter l'intérêt porté par les intellectuels à la question oral/écrit, à Jean Jacques Rousseau². La synthèse des deux images est représentée par l'enfant sauvage. Chaque société en a découvert son spécimen archétypal: dans les bois, élevé par les loups, à l'antithèse du citadin à la mallette. Un objet de musée.

1. Le thème et la critique de la réduction idéologique des faits de culture ou d'histoire à des faits de nature, à des «phénomènes naturels», ont été particulièrement développés par Claude Levi Strauss aux débuts de son oeuvre -void Race et histoire- puis maintes fois repris dans la littérature anthropologique.

2. Havelock, Eric (1986), The Muse Learns to Write, Yale University Press, New Haven. 
L'analphabétisme déborde encore du domaine médical et du domaine ethnographique: il touche au sacré. Non pas dans la version apprivoisée que nous connaissons du sacré (les églises, les religions écrites), mais dans sa version primitive: la magie. Il est relégué au domaine des ombres, il représente un danger, il menace du Mal. Il est à la fois attirant et repoussant. De son innocence, on a peur. Il faut s'en protéger: d'où les campagnes d'éradication, les croisades d'alphabétisation et autres missions d'exorcisation du mal.

Ce phénomène a les caractéristiques du mythe. Roland Barthes comprenait le mythe comme la transmutation magique, imaginaire, de l'histoire en nature, ou de l'existence à l'essence comme disaient les phénoménologues. Dans le cercle mythique, les choses ont la clarté des essences: elles semblent signifier toutes seules. Il n'y a plus de contradiction: le monde a refait son unité. Les événements sont remplacés par des avènements: ils passent au surnaturel. Le mythe est actif. Doté d'une puissance surhumaine, il se substitue a l'historie quand celle-ci est trouble et qu'elle inquiète. Il comble les espaces d'indétermination, il refait l'unité linéaire du temps quand celui-ci apparaît chaotique.

L'analphabétisme est doté de la puissance mythique qu'est l'omniprésence. On l'a découvert à l'action dans tous les secteurs de la société:

- dans l'économie: il serait cause de pertes importantes dans la production, de la faible productivité et de la mauvaise qualité des produits, d'accidents du travail, etc.;

- dans le secteur social: il est associé à la hausse de la criminalité, à la maladie et aux accidents, à l'exclusion croissante de nouvelles couches de population, de plus en plus jeunes;

- dans le domaine socio-politique: il menace directement la démocratie, la sécurité nationale, l'unité nationale. Les analphabètes forment une nouvelle «classe dangereuse»;

- dans la culture: le capital culturel de la nation est menacé de dégradation du fait de la dévalorisation de l'écrit, de la crise de l'école, de l'acculturation ratée des immigrants, de la concurrence de valeurs importées;

- dans la vie privée: les individus et les familles analphabètes présentent une série de troubles psychiques qui affectent leur identité, perturbent leurs rôles, conduisent à l'isolement et à des comportements d'échec, d'agressivité, etc.

Des sondages réalisés dans plusieurs pays confirment que l'analphabétisme ou l'illettrisme est perçu comme un fléau national. Aux Etats-Unis, par exemple, il figurait tout récemment au troisième rang, après la drogue et le sida. Au Canada, au deuxième rang après l'alcool au volant. Les résultats d'enquêtes nationales confortent la certitude de l'existence et de l'étendue du mal. On est face à un phénomène de masses, et non plus de minorités comme c'était le cas il y a une dizaine d'années: 60 millions aux EtatsUnis, un adulte sur 4 au Canada, un sur 5 ou 6 en Europe du Centre, des chiffres sûrement plus élevés en Europe du Sud. Si l'on n'en a pas encore pour l'Europe de l'Est, on peut s'attendre à ce que certains pays mettent bientôt l'alcoolisme, la débacle économique et les désordres sociopolitiques au compte de l'illettrisme, une réalité occultée par les anciens régimes. Seraient épargnés: les pays du nord de l'Europe et le Japon? La Suède, protégée depuis la Réforme par la religion du Livre qui aurait uni, dans la conjuration du mal, l'Eglise, l'Etat et la famille; le Japon, par on ne sait quelle grâce, ou bien parce qu'on n'y a pas fait les mêmes enquêtes.

Le sujet fascine, les chiffres montent. L'illettrisme, qu'on nomme aussi nouvel analphabétisme ou analphabétisme secondaire en Europe, analphabétisme fonctionnel en Amérique du Nord, atteindrait la majorité silencieuse des téléspectateurs et même, selon 
Richard Hoggart, 90\% de la population des pays industrialisés qui n'auraient appris à lire, comme disait Caliban à Prospero dans «La tempête» de Shakespeare, que pour savoir mieux jurer ${ }^{3}$.

L'archéologie et l'histoire comparée du mythe contemporain de l'analphabétisme sont à faire. Le travail est entrepris par différents chercheurs dans plusieurs disciplines (par exemple: Harvey Graff, The Literacy myth, 1979). Les questions qui nous intéressent maintenant sont de deux ordres:

- la déconstruction du mythe: quand, comment, par qui et pourquoi il a été créé? Où en le sert et à quoi il sert? «Pourquoi cette maladie là fut-elle inventée?», demandent Jean-François Lae et Patrice Noisette. «Pourquoi les médecins sont ils si nombreux à accourir, si prolixes sur leur traitement, et si muets sur les effets de ces traitements?» ${ }^{4}$.

- la reconstruction des faits, par l'exploration des problèmes occultés par le mythe. Des problèmes de nature méthodologique, mais aussi d'éthique et d'action: les définitions de l'objet ou des objets d'enquête et leur mesure, la dichotomie analphabétisme/alphabétisme qui pose des problèmes éthiques outre ceux de taxinomie, la question des priorités d'action, en tenant compte des «raisons d'Etat», des demandes de la société civile, des attentes et des droits des individus.

Le champ de ces questions est immense, surtout d'un point de vue international. Il suffira ici d'en expliciter certaines et d'indiquer des pistes de travail.

\section{LA DECONSTRUCTION DU MYTHE}

Le mythe de l'analphabétisme et de sa salvation par l'alphabétisation universelle s'est construit par occultation de l'histoire. Sa déconstruction appelle la recherche historiographique sur l'histoire contemporaire, qui commencerait après la dernière guerre mondiale. Il semble en effet que le problème moderne de l'analphabétisme de masses ait été publiquement posé aux Etats-Unis, pendant cette guerre, par le Président Roosevelt. Et peu de temps après la guerre est fondé l'UNESCO (1947), avec la mission de combattre l'analphabétisme dans le monde. Il est remarquable que cette mission, dirigée vers. le tiers-monde, ait rapidement (1956) conceptualisé ses interventions à l'aide d'une notion de création et d'usage américains: l'alphabétisation fonctionnelle...

Kenneth Levine remarque que, jusqu' au milieu des années 60, l'orthodoxie officielle consistait à parler de l'analphabétisme en terme d'épidémie, comme la malaria; on en faisait une sorte de pathologie culturelle susceptible d'éradication complète au moyen d'un traitement standard par l'éducation: celui que l'on appliquait dans les pays du Nord, soit l'éducation primaire universelle ${ }^{5}$.

Le discours sur l'analphabétisme, de même sa mesure (recensement de 1947 aux USA), seraient donc déjà vieux d'un demi siècle. Un mythe crée l'illusion du nouveau.

3. Hoggart, Richard (1992), «Literacy and the Crisis in Europe Today (unofficial summary by Leslie Limage, Unesco), Literacy and Basic Education on the Eve of the 21st Century, Unesco/Council of Europe, Hamburg/Strasbourg.

4. Lae, Jean François, Noisette, Patrice (1985), Je, tu, il, elle apprend, La Documentation française, Paris.

5. Levine, Kenneth (1986), The Social Context of Literacy, Routledge, Kegan Paul, London. 
Quand il vieillit et qu'il s'érode, il faut renouveler les rituels, les célébrations et les discours. Ce qui fut fait dans les années 80, avec la consécration symbolique, en 1990, de l'Année internationale de l'alphabétisation. Mais pourquoi cette recréation, dans la même symbolique?

Eric Havelock indique une piste féconde, tirée de son expérience qui semble avoir été partagée par d'autres intellectuels éminents de son époque. Il situe le nouvel intérêt porté par la communauté intellectuelle occidentale à la question des rapports entre l'écrit et l'oral, à l'après-guerre. Un événement particulier aurait ouvert de terrain de recherche, depuis très fécond: l'utilisation massive de la radio pendant la guerre, en particulier pour capter les messages d'Hitler.

On a ainsi redécouvert la puissance de l'oralité médiatisée par de nouvelles technologies de communication. L'expérience aurait été fascinante; elle a fait peur. Et l'une des questions qu'elle a suscitée depuis concerne la modernité de l'écrit, son avenir dans les communications, les effets de son déclin appréhendé pour la culture écrite, les dangers pour 1'humanité. M. McLuham (La Galaxie Gutemberg), C. Lévi-Strauss (La pensée sauvage), J. Goody (La raison graphique), W. Ong (Orality and Literacy) ont ouvert la voix à ces recherches, contemporaines des premières campagnes d'alphabétisation.

La première guerre mondiale, par le courrier, la poste, les journaux populaires et les propagandes nationales écrites, avait consacré l'importance de l'écriture. La deuxiéme aurait signalé son déclin possible, un changement culturel fort menaçant puisque dans chaque territoire l'ordre national avait jusqu'alors été maintenu par la culture écrite et les institutions qui ont pour fonction de la préserver, de la valoriser, de la transmettre. La radio, comme la télévision, la téléphone, la micro-informatique aujourd'hui signalent des changements profonds dans le modes de communication d'une époque dont ils ne sont pas, évidemment, la seule cause. Mais ces signaux prennent valeur de symboles puisqu'ils menacent l'ordre écrit: par la technologie, un saut aventureux dans l'inconnu (G. Orwell: 1984) ou par le retour à l'oralité, avec ses connotations primitives catastrophiques (un milliard d'analphabètes adultes dans le monde, «l'analphabétisme de retour» dans les pays industrialisés).

Dans cette recherche des origines du mythe de l'analphabétisme, il faudrait remonter beaucoup plus loin. On en trouve, par exemple, une trace «moderne» déjà en 1855 en Nouvelle Angleterre, où l'on aurait commencé à mesurer le niveau d'alphabétisme de la population à cause de la forte immigration irlandaise, catholique et analphabète, menaçante pour l'ordre établi. L'usage des termes «literacy» et «illiteracy» daterait de cette époque et de ces événements. (Il serait utile de faire l'historiographie comparée des premiers usages du terme dans différents contextes socio-linguistiques).

Quels discours, quels groupes sociaux ont le plus contribué à la construction de l'analphabétisme tel qu'on en parle actuellement? Chaque configuration nationale est particulière. Mais dans une coupe transnationale, on peut identifier des groupes-clé:

- une fraction du milieu scientifique, en particulier des psychologues de l'apprentissage, des spécialistes de la lecture, des docimologues, des sociologues. Ils ont fourni des justifications scientifiques à la théorie du déficit individuel ou culturel;

- les institutions et les réseaux spécialisés dans l'alphabétisation: l'UNESCO, les associations nationales et internationales de volontaires dans la lutte contre l'illettrisme, des organisations gouvernementales; 
- le milieu scolaire, depuis l'identification de l'échec scolaire et ses traitements spéciaux; également le milieu de l'éducation des adultes, de la formation de base à la formation professionnelle;

- des groupes de pression du milieu des affaires (papier, livre, imprimerie), des industries culturelles, des services de l'écrit, de la presse;

- des intellectuels-écrivains qui ont trouvé leur vocation et leur public dans la lutte contre l'illettrisme, la dénonciation du déclin culturel national, etc.;

- des politiciens, des hommes et femmes d'Etat qui on fait passer le sujet dans les législations et les priorités nationales;

- des militants et groupes d'action culturelle (contre-culturelle), de défense des droits de minorités;

- le milieu du travail social, surtout sa frange associative, souvent en liaison avec le groupe précedent;

- des journalistes, réalisateurs, professionnels de l'information écrite et parlée... J'en oublie sûrement.

Ces groupes et individus, séparément, ont produit des discours fort différents, parfois contradictoires et opposés. Mais ils se sont regroupés en associations et en coalitions, ils ont participé aux mêmes événements, ils ont été rassembles dans des mouvements nationaux et supranationaux qui ont pour effet de neutraliser les oppositions et d'absorber les différences. Ils ont construit un discours syncrétique de l'analphabétisme qui réalise le consensus dans la mission à entreprendre. Un macrodiscours qu'un public grandissant attend de ses idéologues populaires et qu'on met en scène publiquement, du local a l'international. Un discours conforté par les images et les témoignages de ces nouveaux acteurs: des analphabètes dans le malheur, en réhabilitation, on hors de danger. Certains se sont découvert un nouveau rôle dans ces témoignages à nu' ${ }^{6}$.

J. F. Lae et P. Noisette, qui ont observé ce mouvement en France, en arrivent à cette question sur la réalité de l'illettrisme: «Un phénomène apparaît nettement: voici que de lieux multiples (mais identifiables) des voix s'élèvent pour dénoncer le «scandale silencieux» de l'illettrisme. Cette concordance bien réelle n'est-elle pas en fin de compte la «définition» la plus précise de la nébuleuse polymorphe de l'illettrisme?»?

Une définition large et relative balise quand même cette «nébuleuse polymorphe» qui rallie pratiquement tous les groupes, celle de l'UNESCO (1951): «est analphabète toute personne incapable de lire et d'écrire, en le comprenant, un exposé simple et bref de faits en rapport avec sa vie quotidienne». Dans les pays industrialisés, on dénomme cette personne analphabète fonctionnelle ou illettrée. La différence permet d'absorber l'objection de la scolarisation universelle dans ces pays. D'autres apports sémantiques, comme le fameux «continuum» de l'alphabétisation, ouvrent tout grand le bassin démographique des analphabètes que les enquêtes vont dénombrer. Il y en aura de plus en plus. Par exemple, au Québec, d'une enquête à l'autre, on est passé de $4 \%$ à $28 \%$ de la population adulte qui aurait de sérieux problèmes en lecture et en écriture. Le discours concerné, qui dit aussi que la société entière est concernée, s'indigne des sous-estimations ${ }^{8}$.

6. Hautecoeur, Jean-Paul (1984), Anonymus Autoportrait, Albert St. Martin, Montréal.

7. Lae, J. F.; Noisette, P., op. cit. 
A la question «pourquoi ce mythe, à quoi il sert?», une première réponse transnationale a été esquissée. Des signes de changement social menacent la culture écrite, l'ordre écrit: dans les technologies de communication, dans l'idéologie (par exemple, le rock: Pink Floyd «The Wall»), dans l'école en crise, dans les conflits de cultures dus à l'immigration ( $« \mathrm{Je}$ ne veux pas lire vos livres, tout est dans le Coran» ${ }^{9}$ ) dans les estimations quotidiennes de la «baisse de niveau» des élèves comme des productions culturelles. Le constat est fait que l'ordre écrit est menacé.

Mais, comme l'a montré Jean Hébrard dans le cas de la France, le diagnostic de crise de la culture n'explique pas encore «l'invention de l'illettrisme» ni les campagnes d'alphabétisation $^{10}$. Pendant les 30 années de croissance, il n'est pas question d'illettrisme en France. Des associations avaient développé et fait connaître l'alphabétisation, mais limitée au public immigré. Dans l'école, l'on avait observé une progression importante de l'échec scolaire et déjà expérimenté des enseignements spéciaux pour les enfants en difficulté d'apprentissage de la lecture. Dans le milieu du sous-prolétariat («Quart-monde»), des associations avaient déjà transformé les services caritatifs en actions culturelles, comprenant l'alphabétisation. Mais toutes ces interventions étaient circonscrites et discrètes.

Dans les années 70, la nouvelle conjoncture de crise économique, de mutations industrielles, de chômage massif, de paupérisation de classes jusqu'alors épargnées («nouveaux pauvres»), etc., a eu pour effet de mettre au premier plan les questions puis l'offre de formation. L'école s'est trouvée interpelée publiquement du fait de l'échec scolaire massif. Les associations ont été mobilisées pour intervenir dans les formations «de bas niveau». Jean Hébrard fait l'hypothèse que l'illettrisme en France est né de la saturation de l'offre de formation à la fin des années 70 . Une analyse similaire est faite pour la Belgique $^{11}$. Elle est aussi globalement applicable au Canada ${ }^{12}$.

La désignation par l'illettrisme ou l'analphabétisme fonctionnel permet de rassembler, en un concept générique, toutes sortes de populations affectées par la crise économique, la crise de l'école, les migrations, le déclassement social et l'exclusion. Elle les canalise toutes vers la formation (par l'offre libérale jusqu'à la contrainte et l'obligation légale). Elle mobilise pour cette opération de grande envergure 1'Etat, l'Eglise, selon le cas, et la société civile par ses réseaux d'associations. Elle refait ainsi le consensus dans la célébration de la valeur de l'écrit que l'école ne parvient plus, à elle seule, à réaliser.

\section{LES DEFINITIONS SOCIALES DE L'ANALPHABETISME}

La critique d'un mythe ouvre la voie au multiple, aux différences et aux contradictions. L'objectivation du mythe de l'analphabétisme découvre des discours concurrents.

8. Hautecoeur, Jean-Paul (1988), «Poids et mesures de l'analphabétisme au Québec», ALPHA 88- Recherches en alphabétisation, sous la direction de J. P. Hautecoeur, ministère de l'Education, Québec.

9. Propos d'un élève rapportés dans le journal Le Monde, 18 octobre 1990.

10. Hébrard, Jean (mai-juin 1990), «Illettrisme, le cas de la France», Actualité de la formation permanente, $\mathrm{n}^{\circ} .106$.

11. Gofinet, Sylvie-Anne; Van Damme, Dirk (1990), Analphabétisme fonctionnel en Belgique, Institut de l'Unesco pour l'Education, Hambourg.

12. Hautecoeur, Jean-Paul (1984), «Essai d'historique de l'alphabétisation et considérations actuelles», ALPHA 84, sous la direction de J. P. Hautecoeur, ministère de l'Education, Québec. 
Une fois décontruit le sens totalitaire qui imposait l'unique comme une évidence, l'analphabétisme devient non plus la, mais les définitions que les groupes sociaux en donnent. Les faits d'analphabétisme sont construits dans des idéologies distinctes (les discours scientifiques en sont), dans les interactions de ces discours et dans leurs changements. Ils sont inséparables des valeurs que les individus et les groupes y investissent ainsi que du prix de l'alphabétisme sur le marché socio-économique. (La conjoncture, on l'a vu, est actuellement très favorable: ces valeurs montent).

Quels sont les principaux points de vue sur les faits dits d'analphabétisme? Quatre lieux sont identifiés: les intellectuels ou les chercheurs, les institutions fortes qui ont l'autorité de définir le sujet, les organisations et les professionnels de l'alphabétisation, les individus et les groupes identifiés comme analphabètes.

Le discours savant sur l'analphabétisme peut schématiquement être séparé en trois: la psychologie des compétences individuelles en lecture et en communication, la sociologie historique de l'alphabétisation, l'anthropologie et la sociolinguistique des pratiques langagières dans différentes cultures. Cette division disciplinaire s'inscrit dans une histoire du discours autorisé sur l'analphabétisme: la psychopatologie de la lecture, discours dominant il y a une vingtaine d'années quand les questions d'analphabétisme étaient liées à l'échec scolaire, a depuis été fortement concurrencée par la sociologie, la linguistique, l'anthropologie et l'histoire qui ont beaucoup élargi la problématique de l'analphabétisme en critiquant, pour la plupart, la réduction clinique à la déficience des capacités de lecture ${ }^{13}$.

Le discours psychologique se développe autour d'un mot clé: la compétence dans les activités de lecture, ou dans la communication par l'écrit. Son lieu d'observation a surtout été, dans l'école, les enfants en difficulté d'apprentissage. Il s'est étendu à l'industrie. Quels sont les compétences requises pour tel type de tâches? Comment sélectionner le personnel idoine? Comment améliorer les rendements dans la lecture ou la communication? Les théories cognitivistes et behavioristes ont donné un fondement scientifique aux diagnostics d'analphabétisme individuel. Leurs applications dans l'école et dans l'industrie ont été largement vulgarisées pour le traitement technique des déficiences ou des déviances mesurées par tests en référence à un modèle normal, moyen ou idéal. Un exemple de cette lecture technique de l'analphabétisme est fourni dans le rapport Des illettrés en France, avec l'échelle des niveaux de lecture ${ }^{14}$.

Le discours sociologique et historique traite de l'analphabétisme en termes d'inégalités sociales et d'acculturation différenciée de l'oral à l'ecrit. Si l'écriture est comprise comme une technologie de la communication, son usage et sa distribution sociale ne sont pas traduits en terme de compétence technique individuelle, mais plutôt d'accessibilité à l'écrit. L'analphabétisme existe à des degrés divers dans les groupes sociaux en marge ou exclus des échanges auxquels participe la majorité de la population. Il n'a de réalité que dans un contexte multidimensionnel de rapports sociaux, économiques et linguistiques qui sont aussi à l'oeuvre dans l'école. On ne peut isoler son «traitement» d'un

13. Barton, David; Hamilton, Mary (1990), Researching Literacy in Industrialized Countries: Trends and Prospects, Institut de l'Unesco pour l'Education, Hambourg.

14. Espérandieu, Veronique; Lion, Antoine; Bénichou, Jean-Pierre (1984), Des illettrés en France, La Documentation française, Paris. 
changement des conditions qui assurent sa reproduction, conditions qu'on nomme généralement exclusion et pauvreté.

Le discours culturaliste tend à refuser l'usage du concept négatif d'analphabétisme. Cet usage caractérise une pensée ethnocentrique et impérialiste («coloniale» comme dit B. Street ${ }^{15}$ ) qui procède par négation de la culture de l'autre pour mieux l'assimiler. A la dichotomie analphabétisme/alphabétisme, on substitue les notions de diversité culturelle et d'usages de l'oralité et de l'écriture. Les pratiques de communication s'inscrivent dans des espaces culturels spécifiques, c'est-à-dire des valeurs et des symboles qui leur donnent du sens, et non pas d'abord sur une échelle de performances. L'efficacité et la fonctionnalité sont aussi symboliques. Par exemple, en milieu populaire comme dans les bandes de jeunes, l'oralité peut s'exercer sur le mode du refus de la culture écrite dominante, comme une valeur affirmative et une marque d'identité. Dans une récente thèse de doctorat au Québec, Hélène Blais substituait le concept d'allographie à celui d'analphabétisme, en proposant de changer de regard pour découvrir d'autres manières de faire ${ }^{16}$.

Ces trois types de discours sont moins exclusifs que complémentaires. Mais ils s'opposent quant à leurs positions socio-politiques. Le discours psychologique se présente comme une démarche expérimentale, neutre par rapport aux valeurs, apolitique. Les discours sociologique et culturaliste s'inscrivent explicitement dans une histoire et dans un contexte de conflits. Ils interviennent pour changer un ordre de discours, de rapports sociaux et de pouvoirs à l'origine du déclassement dans l'analphabétisme. Est-ce qu'il existe des compétences linguistiques qui seraient indépendantes de l'histoire et du contexte social d'un individu? Est-ce que l'analphabétisme est autre chose qu'un fait historique, donc changeant, autre chose qu'une construction sociale?

Un deuxième lieu de définition de l'analphabétisme se distribue principalement entre trois secteurs sociaux: l'école, l'industrie et le commerce, le secteur culturel. Les deux premiers sont puissamment normatifs. L'on mesure les niveaux d'alphabétisme et l'on classe les individus suivant les critères, les valeurs, les exercises scolaires. L'alphabétisation est obligatoire, même après l'âge scolaire. L'idéologie de la «deuxième chance» dissimule le recommencement inévitable. Il faut savoir lire et écrire. Si l'on n'y parvient pas, il faut y croire. L'une des fonctions de l'école est d'inculquer l'idéologie de l'alphabétisation sur l'ensemble d'un territoire national.

Le secteur industriel et commercial, pour ce dernier tout particulièrement dans le marché du livre et de la presse écrite, renforce l'idéologie de l'alphabétisation salvatrice. Mais il définit à ses propres fins -la productivité et le profit- les compétences d'alphabétisation en termes techniques de capacités fonctionnelles. L'analphabétisme est présenté, dans l'industrie, comme un processus de déclassement technologique. Cette idéologie techniciste cache une fonction-clé des épreuves de compétence écrite dans l'industrie: sélectionner le personnel suivant les valeurs culturelles de l'entreprise.

Des intellectuels (écrivains, journalistes) ont aussi un rôle-clé dans la définition sociale de l'analphabétisme. Ils peuvent intervenir pour renforcer les deux discours précé-

15. Street, Brian (1987), «Literacy and Social Change: The Significance of Social Context in the Development of Literacy Programmes», Daniel Wagner ed., The Future of Literacy in a Changing World, Pergamon Press, Oxford.

16. Blais, Hélène (1990), Pour migrer de l'analphabétisme vers l'allographie, thèse de doctorat en sémiologie, Université du Québec à Montréal. 
dents, ou pour critiquer, d'un point de vue élitiste (par ex. R. Hoggart et H. M. Enzensberger), la culture de masses, ou encore pour critiquer élitisme et culture dominante, générateurs d'analphabétisme structurel.

L'école, l'industrie et leurs idéologues affiliés font campagne pour l'alphabétisation, supportés, quand leurs voix sont suffisamment fortes, par le pouvoir politique. Des discours sectoriels sont fondus en un discours national unitaire, aux connotations technocratiques, populistes, caritatives, etc. Campagnes d'alphabétisation ou luttes contre l'illettrisme sont ainsi institutionnalisées, voire légalisées.

Le milieu professionnel et volontaire de l'alphabétisation est hétérogène. Les discours généreux qu'il tient sur l'analphabétisme reproduisent en partie ou en tout les discours déjà évoqués. Certaines organisations font pourtant des évaluations critiques de leur public et de leurs activités d'alphabétisation, en contraste avec les idées reçues. En voice deux exemples, extraits des expériences de PLAN aux Etats-Unis («Push Literacy Action Now») et de La Boîte à lettres au Québec:

«Le discours actuel laisse croire que le problème a une solution... La réalité sous-jacente est fort différente:

- le nombre total d'adultes sous-scolarisés que rattrapent chaque année tous les programmes ne dépasse pas 10 millions;

- plus de 50\% de ces gens s'échappent du filet dans une période de 6 mois à un an, trop peu de temps pour apprendre véritablement quoi que ce soit;

- les personnes inscrites aux programmes ne représentent généralement guère la population sous-scolarisée dans son ensemble...

Très peu de données viennent étayer les soit disant "resultats positifs" de ces programmes... Celles qui existent ne sont guère encourageantes...

Remettre en question et en doute les méthodes de dénombrement des "analphabètes", la définition de l'analphabétisme, l'utilisation des résultats des études et la réaction des EtatsUnis à leur soit disant "crise" ne veut pas dire nier le fait qu'il existe aux Etats-Unis des millions d'adultes sous-scolarisés qui ont besoin de notre aide... Réduire le problème à un manque de connaissances en lecture et en écriture simplifie à outrance le problème complexe et tend à imposer des solutions fondées sur la notion “d'apprendre aux gens à lire et écrire»" 17 .

«- L'alphabétisation de jeunes est une tâche extrêmement difficile... Aucun jeune n'a quitté la Boîte à lettres en sachant lire et écrire convenablement...

- Les jeunes ne sont pas faciles à recruter et ils abandonnent facilement le processus d'alphabétisation. Pour un jeune qui s'alphabétise, il y en a peut-être sept ou davantage qui s'y refusent... - Les jeunes analphabètes ont souvent des besoins urgents à combler autres que celui de l'alphabétisation... Les deux besoins les plus importants demeurent le travail et les relations sociales et amoureuses (comme pour nous tous d'ailleurs)...

Nous dispensons un savoir que plusieurs ne jugent pas indispensable à leur vie, un savoir relégué aux oubliettes par d'autres besoin plus concrets, un savoir qui se transmet lentement et difficilement au-del'a de certaines barrières culturelles...» ${ }^{18}$.

17. Baker, Catherine; Fox, Mike (1990), «L'analphabétisme aux Etats-Unis: discours, recettes et réalités», ALPHA 90-Recherches en alphabétisation, sous la direction de J. P. Hautecoeur, ministère de l'Education/Institut de l'Unesco pour l'Education, Québec, Hambourg.

18. Roy, Sylvie (1992), «Travailler auprès de jeunes analphabètes à la Boîte à lettres», ALPHA 92-Recherches en alphabétisation, sous la direction de J. P. Hautecoeur, Ministère de l'Education. Institut de l'Unesco pour l'Education, Québec, Hambourg. 
Un dernier témoignage, de l'expérience de L'Alpha Mons-Borinage, en Belgique, attaque de front l'attribut d'analphabétisme comme une construction des professionnels de l'alphabétisation:

«Sur le plan quantitatif, nous touchons au total peu de personnes et même la majorité des personnes que nous pourrions croire concernées par notre action s'en désintéresse totalement. Pour le dire crûment, les "analphabètes" ne veulent pas être alphabétisés. Ce n'es pas leur projet, c'es le nôtre. Et ceux que nous touchons le moins, ce sont précisément ceux qui devraient, selon nous, être prioritairement touchés (les plus exlus). Sur le plan qualitatif, l'offre d'alphabétisation comporte un regard négatif sur le public concerné. Les gens sont définis par un manque alors qu'ils sont riches de culture et d'expériences, de réflexion et de rêves. Même si leur vie, leur culture est tronquée, marquée par l'exploitation, l'exclusion, leur réalité est complexe comme toute réalité humaine, ils ont une histoire dont ils sont eux-mêmes responsables. Le regard négatif induit par la démarche d'alphabétisation peut maintenir et renforcer l'exclusion qu'elle prétend éliminer ou diminuer» 19 .

Plus on s'approche de la réalité présumée de l'analphabétisme, plus cette réalité apparaît complexe, problématique, et même étrangère aux gens ainsi qu'aux milieux touchés. Plus il apparaît aussi que l'alphabétisation n'est pas d'évidence la solution à des problèmes qui, s'ils existent par comparaison avec d'autres pratiques de l'écrit, ou bien ne sont pas prioritaires, ou carrément n'existent pas pour les individus à qui on les attribue.

Le dernier groupement duquel on pourrait attendre un discours sur l'analphabétisme, ce sont les «analphabètes». Or ils n'existent pas comme groupe constitué, à part comme clientèle de l'alphabétisation. L'analphabétisme n'est pas une identité pour soi. L'attribut est si mèprisant qu'on ne s'y reconnaît pas naturellement, même si l'on présente le «symptôme» qui semble faire l'accord minimal des spécialistes: des difficultés à lire et à écrire! Il faut encore avoir fait l'expérience et reconnaître que ces difficultés ont été dommageables. Il faut accorder un minimum de valeur à l'écrit. Il faut vivre dans un environnement où l'écrit exige plus que le décodage fortuit des messages ordinaires (qu'on nomme parfois l'alphabétisme de survie). Ou il faut se retrouver par choix, par contrainte ou par obligation dans une institution d'alphabétisation avec l'étiquette «analphabète», dans un groupe fort disparate dont le seul point commun est cette carence abstraite dans l'écrit attribuée par l'institution à sa clientèle.

Il arrive que des individus s'y reconnaissent, incorporent ce nouveau statut, le représentent volontiers devant les autres. Ceux-là sont réellement devenus des «analphabètes». Il y en a qui jouent ce rôle au passé, une fois qu'ils ont été réhabilités. Leurs «success stories» sont en demande. Il y en a qui refusent l'étiquette et le statut, alors souvent on ne les voit plus. Ces derniers sont répertoriés par l'institution d'alphabétisation comme des cas d' «abandon», un comportement typique semble-t-il des «analphabètes non motivés». Se trouve ainsi reconfirmée une déchéance qu'ils ont manifestement refusée.

L'ensemble schématiquement décrit des définitions sociales de l'analphabétisme est plus que différencié, il est contradictoire. La réalité telle que nommée à partir de diver-

19. Arrijs, Omer, «L'expérience de l'Alpha Mons-Borinage», ALPHA 92-Recherches en alphabetisation, op. cit. 
ses positions sociales est plus que complexe, elle est paradoxale. L'analphabétisme est décrit comme un problème majeur par les groupes les plus influents. Il s'impose statistiquement comme un fait massif dans beaucoup de pays industrialisés. Il a justifié la création de dispositifs importants d'alphabétisation ou de lutte contre l'illettrisme. Mais on cherche encore les analphabètes ${ }^{20}$.

Leur principale caractérisque pourrait bien être dans le refus du déclassement ainsi que dans le refus des fins que le déclassement vise: l'alphabétisation obligatoire. En effet, dans certains pays, la législation sanctionne négativement l'abstention ou le refus de participation à la formation qui leur est «offerte».

\section{LES CONTEXTES D'ALPHABETISME}

La critique de la construction sociale de l'analphabétisme ne conduit pas nécéssairement à la négation radicale de la «chose» comme sont tentés de la faire certains ethnologues, ni à son éloge littéraire ${ }^{21}$. D'un point de vue d'action, elle aide à concevoir et à expérimenter d'autres stratégies d'alphabétisation ou d'action culturelle, moins centrées sur les exercices de lecture, d'écriture et de calcul, plus autonomes par rapport à l'école, orientées vers la solution pratique des problèmes quotidiens prioritaires qui passent rarement par le long apprentissage de l'écrit, et mieux intégrées dans les organisations communautaires locales. Ces actions supposent la participation volontaire des intéressés, une condition seulement possible quand des individus ne sont plus fichés comme «analphabètes».

Du point de vue de la recherche, la critique de l'analphabétisme a fait déplacer la question. La notion est trop chargée, trop active, éthiquement trop négative pour être restaurée. Elle n'aide pas à penser ni à changer l'action. Il faut aussi explorer la question de l'alphabétisme autrement que par les seules idéologies. Comment objectiver les interactions avec l'écrit dans un contexte donné de communication, défini par un ensemble de variables techniques, psychologiques, sociologiques, économiques, culturelles ou symboliques? Quels sont les rapports, subjectifs et objectifs, des individus et des groupes aux moyens de communication auxquels ils ont accès dans une situation précise?

Il y a deux entrées à ces questions, comme l'a suggéré J. Goody en transformant le concept marxiste de mode de production en mode de communication: une variable matérielle ou technologique, et une variable sociologique: les rapports de communication, ou les usages individuels et collectifs de l'information ${ }^{22}$. En empruntant ces concepts utiles au marxisme, il faut encore se délester d'au moins deux de leurs prémisses méthodologiques: le matérialisme qui ferait des moyens de communication la variable toujours déterminante, et l'économisme qui évacue trop facilement les échanges symboliques et les définitions culturelles des valeurs.

20. Hautecoeur, Jean-Paul, «Offre généreuse, demande bloquée: le paradoxe actuel de l'alphabétisation», ALPHA 90, op. cit.

21. Magnus Enzensberger, Hans (march 1987), «In praise of the illiterate», Adult Education and Development, 28.

22. Goody, Jack (1979), La raison graphique, Editions de Minuit, Paris. 
Quelles sont les principales variables à interroger dans l'analyse d'un contexte social de communications?

- Le développement des technologies de communication: la distinction est souvent faite entre alphabétisme primaire («basic literacy») et alphabétisme informatique, comme s'il s'agissait de deux niveaux différents d'acculturation à l'écrit. En réalité, la plupart des objets et des situations qui impliquent l'écrit, et souvent l'oral, sont médiatisés par l'électronique. La majorité de la population est, au moins passivement, alphabétisée au moyens de messages électroniques. Quels changements culturels profonds la «révolution informatique» a opérés dans les façons de penser et de communiquer?

- L'économie ou le marché des biens de communication: la question se pose pour l'électronique, pour le papier, pour les services de la communication à distance. Est-ce que ces biens dans telle ou telle région sont rares, courants, universalisés? Comment ils se distribuent? Est-ce que leur production augmente ou diminue? Est-ce qu'ils sont gratuits? (Par exemple, les journaux de quartier gratuits, ou l'information téléphonique). Quelle place occupent les industries culturelles dans l'économie locale?

- La consommation des messages, des objets, des outils et des services de communication: soit la partie demande en rapport avec l'offre précédente d'un point de vue économique. Quels types et quelle quantité de biens écrits médiatisés par l'écrit, entrent dans un foyer? En sortent? A quelle fréquence? Quelle classe de population achète un micro-ordinateur? Qui écrit et reçoit des lettres?

- Le contrôle politique du marché. $\mathrm{R}$. Hoggart et $\mathrm{K}$. Levine ont particulièrement insisté sur cet aspect: la politique de distribution et la censure des biens culturels, la «qualité» des produits de culture de masses, le rôle de l'Etat dans la presse, la monopolisation de l'information par le secteur privé ou par l'Etat. Ces questions se posent en régimes totalitaires comme en régimes d'économie libérale. Quelles sont les possibilités réelles de recevoir tel type de message? Quelles sont les chances réelles de pouvoir en émettre? Dans beaucoup de situations, le contrôle de l'information a pour effet une alphabétisation restreinte, souvent limitée à la réception ou à la lecture, et bloquée dans l'expression, l'échange, le dialogue.

- La politique de transmission culturelle, en particulier par l'école. La question de l'accessibilité de droit aux services scolaires et aux services d'éducation continue ne se pose pratiquement plus dans les pays du Nord. Mais se pose avec véhémence celle de la qualité des services pour différentes populations; de la sélectivité des pédagogies, des institutions, des filières; de la programmation idéologique des textes scolaires et de la formation des enseignants. Olga Kunst Gnamus posait ainsi la question en Yougoslavie: «Comment passer du modèle d'alphabétisation prescrit qui transforme la sphère culturelle en un itinéraire sans joie au concept de littérisme réfléchi, critique et créatif au service d'une société démocratique où homogénéité et hiérarchie font place à hétérogénéité, dialogue entre les différences et communication interculturelle?» ${ }^{23}$.

- Les valeurs attachées à l'écrit, à l'alphabétisme: il s'agit de valeurs d'échange, par exemple sur le marché de l'emploi, qui déterminent la position socio-économique; aussi de valeurs d'usage, dans les rapports pratiques et affectifs que les individus et les

23. Kunst Gnamus, Olga, «Literacy at the Threshold of the 21st Century», Literacy and Basic Education in Europe on the Eve of the 21st Century, op. cit. 
groupes ont avec l'écrit. Les institutions, mais aussi l'état du marché de la main d'oeuvre, décident du prix des premières. K. Levine parle ainsi du «recul des objectifs de l'alphabétisation. En raison du prestige que confère l'instruction, toute amélioration générale des normes en la matière désavantagera automatiquement les groupes les moins instruits» ${ }^{24}$. La valeur d'usage varie suivant les besoins personnels et les désirs, culturellement transmis, attachés aux objets écrits.

- Les pratiques ou les usages de l'écrit: quand, comment, pour quels usages on se sert de l'écrit, de l'oral, des deux, du crayon ou de l'imprimante dans la vie quotidienne? Comment on résoud concrètement un problème d'information quand il manque des éléments au contexte? Par exemple, en mettant à contribution la communauté au lieu d'un seul acteur ou par la division sexuelle des actes de communication: 1'écriture aux femmes, les comptes et la signature aux hommes, etc. David Barton insiste particulièrement sur l'importance de l'observation des «événements» de lecture ou/et d'écriture dans le quotidien, sur la distinction des «domaines» d'usage de l'écrit, sur les «pratiques» culturelles distinctes des groupes sociaux à l'occasion de tel événement, et sur les valeurs attachées aux actes, ou les sentiments qui s'y investissent ${ }^{25}$.

- Les idéologies qui définissent un sens et une valeur à l'alphabétisme: le sujet a été traité dans les parties précédentes. En font partie les discours scientifiques qui définissent d'autorité le sujet, le plus souvent par occultation de leurs fonctions idéologiques et corporatistes.

- La diversité linguistique, la diversité culturelle: ces variables ont déjà été croisées par les variables politiques. Suivant le contexte, on est en présence de plusieurs langues, de langues écrites et de langues orales, de langues officielles et de dialectes, de plusieurs domaines linguistiques, etc. En fait, il est rare qu'un contexte culturel soit linguistiquement homogène, alors que les normes sociales et institutionnelles imposent généralement l'unilinguisme. Cette question du rapport de force entre langues s'applique aussi entre langues naturelles et langues programmées. Celles-ci peuvent coexister ou s'exclure. Les derniers travaux de Paulo Freire et ses collaborateurs aux Etats-Unis abordent ces questions d'hégémonie linguistique et culturelle dans la normalisation de l'alphabétisme au singulier ${ }^{26}$.

- Enfin et évidemment, il y a cette variable de la compétence individuelle dans l'interaction physiologique et psychologique avec l'écrit. C'est celle qui est traitée le plus abondamment dans la littérature de l'alphabétisation. C'est aussi celle qu'on cherche le plus souvent à mesurer et pour laquelle il existe déjà un matériel méthodologique important.

David Harman comparait les recherches actuelles sur la compétence à la quête du Saint Graal: au singulier, abstraite du contexte culturel, du sens et de la valeur que les individus lui reconnaissent, on ne la trouvera pas $^{27}$. Elle n'est qu'une variable dans un

24. Levine, Kenneth (1990), «Le futur de l'alphabétisation et l'alphabétisation du futur», Alphabétiser? Parlons-en!, Bureau international de l'Education, Genève.

25. Barton, David, «Comprendre l'alphabétisme au quotidien», ALPHA 90, op. cit.

26. Freire, Paolo; Macedo, Donaldo (1987), Literacy; Reading The Word and The World, Routledge-Kegan Paul, London.

27. Harman, David (1987), Illiteracy, a National Dilemma, The Adult Education Cie, Cambridge, USA. 
contexte lui aussi changeant et multiple. Dans certains cas, elle peut être déterminante: par exemple les, cas d'autisme ou de paralysie cérébrale où il n'y a plus de communication. Mais même dans une situation de non-alphabétisme intégral où l'écrit est carrément étranger aux échanges vernaculaires, on trouvera un usage aux objets écrits, on saura les introduire dans les circuits de la communication en les dotant de sens et de valeur propres au nouveau contexte. C'est ce qu'il faut trouver: le sens, la valeur et les usages de l'écrit dans un contexte culturel particulier.

La compétence alphabétique n'est pas non plus une capacité unique, limitée au champ linguistique et dans celui-ci à une activité particulière comme la lecture. On exclut souvent les échanges oraux de l'alphabétisme, parfois l'écriture, même le silence (Il y a un mot en anglais pour désigner l'absence de pratique de l'écrit: «aliteracy». En français, on désigne ces personnes comme des non-lecteurs, qui peut être synonyme d'illettrés). Pourquoi la fixation sur la lecture, et non pas, par exemple, sur l'expression personnelle écrite, orale et silencieuse?

Dans les enquêtes récentes, les tests de lecture ont tendance à être remplacés par des simulations de résolution de problèmes qui font appel à des capacités diverses et à des registres différents de savoir-faire ${ }^{28}$. Ces épreuves (imposées, même en douceur) mettent à contribution l'expérience acquise dans le contexte familier de l'individu, le savoir pratique et le sens commun à ce contexte. Certaines épreuves complexes peuvent n'exiger qu'une compétence élémentaire en lecture (par exemple, quand les questions sont posées oralement et qu'il suffit, pour y répondre, de reconnaître globalement quelques signes iconiques ou symboliques). Et encore! Dans la vie quotidienne, le décodage et la réponse n'imposent pas qu'on soit seul: on s'arrange souvent mieux à deux ou à plusieurs.

Le fameux continuum de l'alphabétisme, qu'on cherche à mesurer sur des échelles de compétence, est une abstraction. Mais c'est une abstraction active, puisqu'elle va imposer ses normes de procédure, de classification, d'interprétation. Dans la situation d'enquête ou d'entrevue par test, il n'y a pas de négociation du sens, de la valeur, du pouvoir. L'échange est forcé. Le contexte créé risque d'être conflictuel, voire antagoniste (Institutions d'enquêtes et personnel scientifique chachent bien ce fait). La véritable épreuve pour les enquêtés est de s'en sortir. Comment vont-ils faire? Voilà la question intéressante devant un tel événement d'alphabétisme.

\section{CONCLUSION}

La recherche et l'action en alphabétisation méritent, au constat des résultats (controversés) d'autant de militantisme, de missionariat et d'activisme dans la bonne cause de la lutte contre l'illettrrisme, qu'on retourne à l'objet de fascination qu'est l'illettrisme ainsi qu'aux mobiles et au fonctionnement de cette singulière mobilisation. L'Année internationale l'aura consacrée et renforcée. Elle aura aussi permis des rencontres qui peuvent être l'occasion d'une interrogation renouvelée sur l'ensemble des faits sociaux syncrétisés dans l'appellation d'alphabétisme (ou son contraire).

28. Satin, Alvin; Kelly, Karen; Montigny, Gilles; Jones, Stan (1990), National Literacy Skill Assessment: The Canadian Experience, Statistique Canada, Ottawa. 
La première intention de ce texte était de relativiser les notions d'illettrisme et d'alphabétisme en les saisissant comme des faits idéologiques à situer dans une histoire qui explique leur émergence et leur accrochage dramatique dans l'imaginaire de beaucoup de sociétés occidentales. A l'encontre des positions positivistes renforcées par les techniques quantitatives de mesure des niveaux de compétence des individus, l'analphabétisme a été interrogé comme un mythe dont la principale caractéristique n'est pas d'être une idée fausse, mais un discours symbolique destiné à agir efficacement dans une histoire en crise. Unitaire, il vise à réaliser un vaste consensus social dans l'identification de problèmes multiples que connaissent les sociétés de l'Ouest, ainsi que dans la conjuration du mal nommé illettrisme ou analphabétisme.

Si l'on perd de vue les fonctions mythiques de la lutte contre l'illettrisme, l'on risque de ne considérer que les objectifs explicités de la formation et de n'en attendre que des résultats pédagogiques. Or ceux-ci sont fort mal connus et des évaluations internes au milieu de l'alphabétisation montrent qu'ils sont douteux. Mais les effets sociaux du discours sur les dangers de l'analphabétisme sont beaucoup plus sensibles: la mobilisation de grande envergure des plans locaux au plan international, la croissance du champ de la formation de base et de l'emploi dans un secteur avant occupé par des associations caritatives et des travailleurs bénévoles, la généralisation de la formation comme alternative contraignante au chômage de longue durée et à l'exclusion.

Le thème porteur de l'alphabétisation est devenu la formation de la main d'oeuvre, potentielle ou en emploi. Cependant, l'occupation formative ne mène que rarement à l'emploi. Elle en est une alternative de longue durée pour une population croissante. Elle a aussi l'avantage de se présenter comme une sortie des programmes d'assistance sociale pour les populations «à risque». Dans quelle mesure y parvient-elle? Ces questions d'évaluation des résultats de l'alphabétisation, en rapport avec ses objectifs pédagogiques explicités mais aussi avec ses fonctions sociales plus diffuses, s'imposent à la recherche qui a, jusqu'à maintenant, surtout investi dans le développement des activités de formation.

La deuxième intention de l'article était de découvrir un autre terrain de recherche aussi peu fréquenté que le précédent: les conditions matérielles et sociales du développement de l'alphabétisme dans un contexte historique particulier, et l'analyse comparée de contextes différents comme peuvent en présenter, par exemple, les pays de l'Est et de l'Ouest de l'Europe. Le terrain dans lequel on a investi le plus, la pédagogie, en est un qui s'inscrit généralement dans le discours normatif de la lutte contre l'illettrisme. D'où la fixation sur le thème de la compétence individuelle en lecture (surtout) et en écriture, un archétype de l'idéologie scolaire.

Il a été proposé d'élargir la problématique de l'alphabétisme à celle des conditions matérielles et des conditions sociales de l'usage des moyens d'information et de communication, à celle des usages différenciés suivant l'héritage culturel et l'appartenance sociale des individus, à celle de l'historiographie comparée de l'alphabétisme, des campagnes d'alphabétisation et de la résistance de certains groupes au contrôle ou à la domination culturelle.

Cet autre programme de recherche peut être particulièrement fécond pour la coopération nouvelle entre l'Est et l'Ouest du fait que l'histoire politique différente des deux régions a crée des conditions fort différentes de développement des communications. $\mathrm{Si}$ la crise économique (ou les crises conjuguées) à l'Ouest est à l'origine de l'invention de 
l'illettrisme et de la priorité donnée à sa résorbtion, à quelles techniques culturelles ont donné lieu les récentes crises des pays de l'Est? Quelles utopies découvre-t-on dans l'alphabétisme? Pour quelles fins individuelles et collectives? Ou encore, la déstructuration du marché de l'emploi et de l'organisation sociale aura-t-elle comme effet la mobilisation pour la formation?

Avant de mettre au point des techniques universelles de mesure des niveaux d'alphabétisme, il serait plus approprié de saisir un tel phénomène diffus dans ses différences dues, moins aux niveaux de compétence individuelle qu'aux contextes sociaux qui leur donnent un sens. 